\title{
Disorders of sex development (DSD): an overview of recent scientific advances
}

\author{
Gerard S. Conway* \\ Department of Endocrinology, University College London Hospital, London, UK
}

(Received 6 June 2012; final version received 25 October 2012)

\begin{abstract}
Developments in biotechnology have radically altered clinical and research themes in the small field of disorders of sex development, as in other rare medical conditions. In the age of genetics, an increasing number of DSDs have been identified. Aided by larger populations of people with DSD diagnoses attending specialist centres, these discoveries are enabling medical doctors and scientists to map out the long-term clinical presentations of a number of conditions. In terms of benefits to patients, the new discoveries have paid dividends in two specific ways. First of all, even though knowledge may remain incomplete, our understanding of the risk of malignancy in retained gonads has improved significantly. Consequently, care providers are in a stronger position than they have ever been to advise patients in their decision-making processes around removing or retaining their gonads. Second, more scientific understanding has led to improved fertility outcomes for women who have a uterus, as in Turner's Syndrome, Congenital adrenal hyperplasia and Swyer's Syndrome.
\end{abstract}

Keywords: Turner syndrome; congenital adrenal hyperplasia; Swyer syndrome; cloacal exstrophy

\section{Introduction}

In recent years, the scientific drive in the field of DSD has focused on identifying new gene variations (mutations) that lead to atypical sex development. There is often then a time lag between such genetic discovery and routine genetic screening with larger populations. Now that automated gene sequencing is available in many biochemistry departments, however, the identification of genetic variations as part of a routine medical work up is becoming more common. The next phase of development of our knowledge base for DSDs will be in identifying subtleties of physical health characteristics and long-term physical health risks of each genetic group.

Some individuals with a DSD diagnosis may find genetic research an esoteric subject with little relevance for their well-being. However, the scientific agenda is often driven by pertinent medical issues. First of all, there are families with several members who have a DSD diagnosis and these families may also have individuals who carry a variant gene or a group of variant genes. This topic is currently especially relevant to those diagnoses that are associated with male-typical XY (sex) chromosomes. Individuals with these diagnoses present to a greater or lesser extent male-typical physical sex characteristics, such as a

*Email: g.conway@ucl.ac.uk 
Table 1. A summary of the new nomenclature relating to disorders of sex development.

\begin{tabular}{ll}
\hline Previous & \multicolumn{1}{c}{ Current } \\
\hline Intersex & Disorder of Sex Development DSD \\
XY sex reversal & 46, XY gonadal dysgenesis \\
Male pseudohermaphrodite & 46, XY DSD \\
Undervirilisated XY male & \\
Female pseudohermaphrodite & 46, XX DSD \\
Masculinised XX female & \\
True hermaphrodite & Ovotesticular DSD \\
XX male, XX sex reversal & $46, X X$ testicular DSD \\
\hline
\end{tabular}

significant amount of facial and body hair, enlargement of the clitoris and deepening of the voice. Second, it may be possible to identify patterns of health concerns that could affect long-term follow-up once we know how to differentiate one condition from another at the genetic level.

Rather than going into the detail of each new genetic variant in the physical sex differentiation pathway, this overview will discuss the common conditions that come under the heading of DSD and then review the advances that affect medical management derived from experience in a tertiary multidisciplinary DSD clinic. Detailed reviews of new genetic variations in the pathway of sex differentiations can be found in several recent papers (Bashamboo, Ledig, Wieacker, Achermann, \& McElreavey, 2010; Ludbrook \& Harley, 2011) and will not be repeated here.

Scientific progress will also be promoted by the increasing popular strategy of collaborating groups of clinicians combining knowledge of larger cohorts of individuals with DSD diagnoses so that long-term outcomes can be documented. As DSDs are rare conditions, it is important to construct larger cohorts so that the spectrum of variability on each condition can be documented. An international collaboration entitled iDSD is one such initiative. This work will allow for greater confidence and speed of diagnosis for new cases as they arise. Table 1 shows the current nomenclature for the classification of DSD. This overview also considers related conditions outside of the current classification including Turner's Syndrome and Klinefelter's Syndrome.

\section{Sex chromosome DSD}

DSD diagnoses triggered by variations in the sex chromosomes (46, XX being female typical and 46,XY male typical) are associated with absent development or early demise of the gonads that would otherwise develop into testes or ovaries. Technical advances in this field include the use of probes to identify the number of $\mathrm{X}$ and $\mathrm{Y}$ chromosomes within 24 hours. For example, XXY is likely to mean Klinefelter's Syndrome. The application of these techniques to identify variations in foetal sex chromosomes by testing amniotic fluid taken from the womb during pregnancy could pose ethical challenges when offering information that could affect parental decision whether or not to continue with the pregnancy. This is especially so when the genetic make-up is not a strong predictor of the developmental outcome. For example, an individual with Turner's Syndrome may enjoy relatively good health with regular ovarian function at puberty, or present serious and multiple health problems across the lifespan.

Another scientific advance in this area relates to the fertility outcomes for this group of conditions. In Turner's syndrome (TS), which affects only females and is caused by 
complete or partial absence of the 2 nd X chromosome, approximately $20 \%$ of the girls can expect to enter puberty as is typical and have preserved ovarian function that may continue for several years. Previously, no medical help could be offered to this group, apart from monitoring the progress of early loss of ovarian function. With improved techniques in egg freezing, it is now possible to harvest eggs for preservation and future use (Balen, Harris, Chambers, \& Picton, 2010; El-Shawarby, Sharif, Conway, Serhal, \& Davies, 2010). For the $80 \%$ of the TS population without ovarian function and therefore no periods ever and for whom hormonal induction of puberty is required, the wider application of egg donation has allowed many more women to conceive. As we have more information now about the health risks of adult women with TS, we are also increasingly aware of the risk of tearing in the wall of the major blood vessel that can lead to rupture of the heart and sudden death during pregnancy. Therefore, for women choosing egg donation, the whole process of fertility for women with TS has to be carefully managed in a multidisciplinary service (Chalas Boissonnas et al., 2011). The case reports may well have overestimated the health risks of conception via egg donation in TS compared to population-based studies which show good obstetric outcomes (Hagman et al., 2011).

For men with Klinefelter's syndrome, a relatively common form of DSD affecting one in 500 men, the most dramatic advance has been in retrieving viable sperm from the testes (Schiff et al., 2005). Until the advent of micro-testicular sperm extraction techniques, there was no available treatment for the usual presentation of absence of sperm in the ejaculate of men diagnosed with Klinefelter's syndrome. Several research groups are now reporting retrieval rate of viable sperm in up to $50 \%$ of individuals using a painstaking technique whereby the testis is opened and the small tubular structures where sperm can be found are individually inspected under a microscope for the presence of sperm (Schiff et al., 2005; Vernaeve et al., 2004). A single sperm thus retrieved can be injected into a single egg which has been harvested in the context of in vitro fertilisation. Replication of this success rate by other units will be required before we know more about fertility outcome.

\section{6,XY DSD}

46,XY DSD covers three main external anatomical presentations: 46, XY gonadal dysgenesis, androgen insensitivity syndrome and testosterone biosynthesis conditions. A variety of rare conditions that come under this heading are listed in genetic reviews cited above.

Women with 46,XY gonadal dysgenesis (Swyer's syndrome) have gonads that are neither testes nor ovaries, because disruption to regular sex differentiation occurs very early on in embryonic life. The women therefore do not experience menstrual periods ever and do not experience the usual pubertal development in adolescence. Furthermore, the gonads have to be removed because of the high-risk of malignancy. Lifelong oestrogen replacement therapy is required to maintain health. Development of the uterus however does take place and there are now increasing reports showing that pregnancy achieved through egg donation is highly successful (Creatsas, Deligeoroglou, Tsimaris, Pantos, \& Kreatsa, 2011; Michala, Goswami, Creighton, \& Conway, 2008; Tulic, Tulic, \& Micic, 2011).

Complete androgen insensitivity syndrome (CAIS) is due to a problem with the androgen reception gene. Individuals with male-typical $(46, \mathrm{XY})$ sex chromosomes do not have receptors to respond to androgens secreted by the testes. When development of maletypical sex characteristics is absent, the external anatomy is female-typical. Internally, the individual has testes and is without a womb or cervix and a smaller than average vagina. Nearly all individuals diagnosed with CAIS are female assigned and identified. 
Our understanding of CAIS has improved by the study of larger cohorts. In particular, it is now recognised that the risk of malignancy of the testes is considerably less for this condition than for other forms of 46,XY DSD (Cools \& Looijenga, 2011). For instance, it is estimated that the risk of gonadal malignancy in CAIS is less than $1 \%$ compared to approximately $25 \%$ for women with Swyer's syndrome (Cools et al., 2005; Pleskacova et al., 2010). This clarification has led to some women with CAIS choosing to retain their testes that are producing steroids to keep them healthy, thereby avoiding the need for longterm oestrogen replacement therapy. It is also noted however that the data on gonadal risk is based on paediatric data and may not hold true for women in an older age group (Deans, Creighton, Liao, \& Conway, 2012). Further research is required to find how we can advise women with CAIS as to how safe it is to avoid gonadectomy.

Until recently, women with a 46,XY DSD diagnosis associated with some male-typical sex characteristics (e.g. ambiguous genitalia at birth) were believed to have partial androgen insensitivity syndrome (PAIS), that is, they are partially receptive to the androgen that stimulate development of male-typical sex characteristics (Inacio et al., 2011). With the wider application of genetic analysis and studies of profiles of steroid in the urine, it is now recognised that only a minority of cases are to do with problems of the androgen receptor gene. Some of these conditions overlap completely with two testosterone biosynthesis conditions: 5 alpha reductase deficiency and 17 hydroxysteroid dehydrogenase deficiency (Berra et al., 2011). Greater understanding of the natural history of each condition can now develop and retrospective assessment might allow for better clinical identification of conditions when they are first presented.

Currently, there is a lot of interest in changes to the NR5A1 (SF1) gene in some 46XY DSD conditions. It is thought that NR5A1 mutation accounts for about $15 \%$ of identified cases of 46,XY DSD. However, it is also becoming clear that there is a spectrum of physical features associated with this genetic variation (Ludbrook \& Harley, 2011).

One of the major areas for future research on all forms of 46XY DSD is the integrity of the germ cells - cells within the ovary or testis that eventually develop into eggs or sperm. As such fertility options might be considered before removal of the gonads to reduce cancer risks. So far, no reports of preservation of germ cells have emerged, but this will be an area to watch as the long-term outcome of well-described conditions become clear.

\section{6, XX DSD}

The most common form of $46, \mathrm{XX}$ DSD is the group of metabolic conditions called congenital adrenal hyperplasia $(\mathrm{CAH})$, for which the medically necessary treatment is with adrenal steroid, a group name for fludrocortisone, hydrocortisone, prednisolone and dexamethasone. $\mathrm{CAH}$ affects both females and males. Once again, the main progress in our understanding of $\mathrm{CAH}$ comes from increasing data from adult cohorts. A major scientific advance has been the improved fertility outcomes for women with CAH. Fertility used to be dismal, but is now essentially much less of a problem as a result of attention to detail in adrenal steroid treatment (Casteras, De Silva, Rumsby, \& Conway, 2009; Hagenfeldt et al., 2008).

While girls and women with CAH may have additional psychological challenges compared to their male counterparts, due to the effects on sex anatomy, there is a major concern of the persistently poor fertility outcomes in men (Reisch et al., 2009). Testicular adrenal rest tissues (TARTs) are small nodules in the testis and cause damage because they displace testicular tissue. Testicular failure is common with only a minority of men having sperm 
counts with fertility potential. Early education and secure transition to adult services will be important in improving management of males with CAH (Falhammar et al., 2012).

\section{Cloacal exstrophy}

Cloacal exstrophy is a rare, highly complex condition and a life threatening condition affecting about 1 in 200,000 live births, with a spectrum of anatomical variations affecting multiple organ systems. Survival rates have improved in recent decades. However, there is much to learn as more individuals survive into adulthood. Surgical management typically involves multiple medically essential operations throughout the life span. In general, the advent of multidisciplinary care has shifted the focus to minimise medically nonessential surgery at young ages so as to keep options open for informed choices later in life. Feedback from older age groups (Reiner \& Gearhart, 2004) has allowed for more accurate prediction of gender orientation. In contrast to earlier emphasis on female gender assignment, there is now a greater openness towards male gender assignment.

\section{Conclusions}

Specialisation of clinical services and improved information have increased our knowledge of the natural history of DSDs. Faster and more extensive biogenetic technology, with the introduction of whole genome sequencing, is predicted to advance our knowledge of genetic changes that lead to certain anatomical outcomes associated with DSD. There are initiatives in a number of countries that aim to develop care pathways for rare conditions, informed by an accelerated user movement. These care pathways will facilitate further audit and research, leading to more molecular discoveries and probably changes to medical treatment and clinical care more generally in the next 10 years.

\section{Notes on contributor}

Professor Conway is a consultant endocrinologist at University College London Hospitals and professor of clinical medicine in the Institute for Women's Health, University College London. Clinical research from his department spans a wide spectrum of reproductive and developmental endocrinology.

\section{References}

Balen, A. H., Harris, S. E., Chambers, E. L., \& Picton, H. M. (2010). Conservation of fertility and oocyte genetics in a young woman with mosaic Turner syndrome. British Journal of Obstetrics \& Gynaecology, 117(2), 238-242.

Bashamboo, A., Ledig, S., Wieacker, P., Achermann, J. C., \& McElreavey, K. (2010). New technologies for the identification of novel genetic markers of disorders of sex development (DSD). Sexual Development, 4(4-5), 213-224.

Berra, M., Williams, E. L., Muroni, B., Creighton, S. M., Honour, J. W., Rumsby, G., \& Conway, G. S. (2011). Recognition of 5alpha-reductase-2 deficiency in an adult female 46XY DSD clinic. European Journal of Endocrinology, 164(6), 1019-1025.

Casteras, A., De Silva, P., Rumsby, G., \& Conway, G. S. (2009). Reassessing fecundity in women with classical congenital adrenal hyperplasia (CAH): Normal pregnancy rate but reduced fertility rate. Clinical Endocrinology (Oxford), 70(6), 833-837.

Chalas Boissonnas, C., Davy, C., Marszalek, A., Duranteau, L., de Ziegler, D., Wolf, J. P., . . Jouannet, P. (2011). Cardiovascular findings in women suffering from Turner syndrome requesting oocyte donation. Human Reproduction, 26(10), 2754-2762.

Cools, M., \& Looijenga, L. H. (2011). Tumor risk and clinical follow-up in patients with disorders of sex development. PediatricEndocrinology Review, 9(Suppl 1), 519-524. 
Cools, M., van Aerde, K., Kersemaekers, A. M., Boter, M., Drop, S. L., Wolffenbuttel, K. P., ... Looijenga, L. H. J. (2005). Morphological and immunohistochemical differences between gonadal maturation delay and early germ cell neoplasia in patients with undervirilization syndromes. Journal of Clinical Endocrinology \& Metabolism, 90(9), 5295-5303.

Creatsas, G., Deligeoroglou, E., Tsimaris, P., Pantos, K., \& Kreatsa, M. (2011). Successful pregnancy in a Swyer syndrome patient with preexisting hypertension. Fertility \& Sterility, 96(2), e83-e85.

Deans, R., Creighton, S. M., Liao, L. M., \& Conway, G. S. (2012). Timing of gonadectomy in adult women with complete androgen insensitivity syndrome (CAIS): Patient preferences and clinical evidence. Clinical Endocrinology (Oxford), 76(6), 894-898.

El-Shawarby, S. A., Sharif, F., Conway, G., Serhal, P., \& Davies, M. (2010). Oocyte cryopreservation after controlled ovarian hyperstimulation in mosaic Turner syndrome: Another fertility preservation option in a dedicated UK clinic. British Journal of Obstetrics \& Gynaecology, 117(2), 234-237.

Falhammar, H., Nystrom, H. F., Ekstrom, U., Granberg, S., Wedell, A., \& Thoren, M. (2012). Fertility, sexuality and testicular adrenal rest tumors in adult males with congenital adrenal hyperplasia. European Journal of Endocrinology, 166(3), 441-449.

Hagenfeldt, K., Janson, P. O., Holmdahl, G., Falhammar, H., Filipsson, H., Frisen, L., ... Nordenskjöld, A. (2008). Fertility and pregnancy outcome in women with congenital adrenal hyperplasia due to 21-hydroxylase deficiency. Human Reproduction, 23(7), 1607-1613.

Hagman, A., Kallen, K., Barrenas, M. L., Landin-Wilhelmsen, K., Hanson, C., Bryman, I., \& Wennerholm, U.-B. (2011). Obstetric outcomes in women with Turner karyotype. Journal of Clinical Endocrinology \& Metabolism, 96(11), 3475-3482.

Inacio, M., Sircili, M. H., Brito, V. N., Domenice, S., Oliveira-Junior, A. A., Arnhold, I. J., . . Mendonca, B. B. (2011). 46,XY DSD due to 17beta-HSD3 deficiency and 5alpha-reductase type 2 deficiency. Advances in Experimental Medical Biology, 707, 9-14.

Ludbrook, L., \& Harley, V. R. (2011). Genetic mechanisms underlying 46,XY DSD with gonadal dysgenesis. Advances in Experimental Medical Biology, 707, 87-88.

Michala, L., Goswami, D., Creighton, S. M., \& Conway, G. S. (2008). Swyer syndrome: Presentation and outcomes. British Journal of Obstetrics \& Gynaecology, 115(6), 737-741.

Pleskacova, J., Hersmus, R., Oosterhuis, J. W., Setyawati, B. A., Faradz, S. M., Cools, M., ... Looijenga, L. H. (2010). Tumor risk in disorders of sex development. Sexual Development, 4(4-5), 259-269.

Reiner, W. G., \& Gearhart, J. P. (2004). Discordant sexual identity in some genetic males with cloacal exstrophy assigned to female sex at birth. New England Journal of Medicine, 350(4), 333-341.

Reisch, N., Flade, L., Scherr, M., Rottenkolber, M., Pedrosa Gil, F., Bidlingmaier, M., . . . Reincke, M. (2009). High prevalence of reduced fecundity in men with congenital adrenal hyperplasia. Journal of Clinical Endocrinology \& Metabolism, 94(5), 1665-1670.

Schiff, J. D., Palermo, G. D., Veeck, L. L., Goldstein, M., Rosenwaks, Z., \& Schlegel, P. N. (2005). Success of testicular sperm extraction [corrected] and intracytoplasmic sperm injection in men with Klinefelter syndrome. Journal of Clinical Endocrinology \& Metabolism, 90(11), $6263-6267$.

Tulic, I., Tulic, L., \& Micic, J. (2011). Pregnancy in patient with Swyer syndrome. Fertility \& Sterility, 95(5), 1789, e1781-1782.

Vernaeve, V., Staessen, C., Verheyen, G., Van Steirteghem, A., Devroey, P., \& Tournaye, H. (2004). Can biological or clinical parameters predict testicular sperm recovery in 47,XXY Klinefelter's syndrome patients? Human Reproduction, 19(5), 1135-1139. 